\title{
Next-generation genome sequencing of a matched normal-tumor pair from a patient with intractable gestational choriocarcinoma: A case report
}

\author{
KAORU NIIMI $^{1}$, EIKO YAMAMOTO ${ }^{1,2}$, SACHI MORITA $^{3}$, MAKI MORIKAWA $^{3}$, HIKARU HATTORI ${ }^{4}$, \\ MIKI HATAKEYAMA ${ }^{3}$, MAMI MORITA ${ }^{3}$, KIMIHIRO NISHINO ${ }^{1}$, YUKARI ODA ${ }^{1}$, ERI WATANABE ${ }^{1}$, \\ TOSHIMICHI YAMAMOTO ${ }^{5}$, HIROAKI KAJIYAMA ${ }^{1}$ and FUMITAKA KIKKAWA ${ }^{1}$ \\ Departments of ${ }^{1}$ Obstetrics and Gynecology, and ${ }^{2}$ Healthcare Administration, \\ Nagoya University Graduate School of Medicine; Departments of ${ }^{3}$ Medical Genomics Center and \\ ${ }^{4}$ Medical Technique, Nagoya University Hospital; ${ }^{5}$ Department of Legal Medicine and Bioethics, \\ Nagoya University Graduate School of Medicine, Nagoya, Aichi 466-8550, Japan
}

Received October 6, 2020; Accepted April 16, 2021

DOI: $10.3892 / \mathrm{mco} .2021 .2305$

\begin{abstract}
Gestational choriocarcinoma is a gestational trophoblastic neoplasia (GTN) originating from trophoblastic cells with abnormal proliferation. Although chemotherapy is effective for treating this cancer, when patients develop chemoresistance, personalized treatment, such as the use of drugs matching their genomes, is required. The present report describes a case of intractable gestational choriocarcinoma identified using a next-generation sequencing (NGS)-based tumor panel. A 51-year-old woman was diagnosed with gestational choriocarcinoma via pathological and short tandem repeat analyses. The patient did not achieve remission despite many regimens of chemotherapy, including high-dose therapy with autologous peripheral blood stem cell transplantation. To identify drugs tailored to this particular choriocarcinoma, NGS was performed on the tumor of the patient, and the tumor genome was compared with that of the patient's blood sample using the NCC Oncopanel System. Consequently, 245 single nucleotide variants (SNVs) with a mean SNV allele frequency of $63.1 \%$ were identified. This high frequency was because the genome of the gestational choriocarcinoma contained part of the genome of the partner. Therefore, our experience of the present intractable case of choriocarcinoma suggested that matched normal-tumor pair analysis is not appropriate for treatment decisions in GTN cases. When using an NGS-based tumor panel to assess choriocarcinoma, researchers must consider whether the genomic DNA of the patient and their partner are involved in the GTN.
\end{abstract}

Correspondence to: Dr Kaoru Niimi, Department of Obstetrics and Gynecology, Nagoya University Graduate School of Medicine, 65 Tsurumai-cho, Showa-ku, Nagoya, Aichi 466-8550, Japan E-mail: kaorun@med.nagoya-u.ac.jp

Key words: next-generation genome sequencing, choriocarcinoma, gestational trophoblastic diseases

\section{Introduction}

Gestational choriocarcinoma is a type of gestational trophoblastic neoplasia (GTN) that originates from trophoblasts and can develop from a normal pregnancy, miscarriage, or molar pregnancy. Its estimated incidence in Japan is 1.9-5.5 cases per 100,000 live births (1). Non-gestational choriocarcinoma shows the same morphological pattern as that of the gestational form but originates mostly from germ cells in the ovary and is rarer and associated with worse outcomes. Short tandem repeat analysis using microsatellite markers is useful for distinguishing gestational choriocarcinoma from non-gestational choriocarcinoma (2).

Chemotherapy is effective for treating gestational choriocarcinoma. However, patients with multiple metastases or metastases to sites other than the lungs often do not achieve complete remission (3). When the cancer develops chemoresistance, more tailored therapies are required, such as drugs selected based on the specific cancer genome. The OncoGuide ${ }^{\mathrm{TM}}$ NCC Oncopanel System (Sysmex Corporation) (4) is a next-generation sequencing (NGS)-based tumor panel that is covered by health insurance in Japan. This panel facilitates the identification of variants of 114 cancer-related genes through matched normal-tumor pair analysis. Here, we report a case of intractable gestational choriocarcinoma identified using this system.

\section{Case report}

A 51-year-old Japanese woman was diagnosed with choriocarcinoma with metastases to the lung, spleen, and lymph nodes. Histopathological examination of the uterine biopsy showed a two-cell pattern of choriocarcinoma, consisting of syncytiotrophoblastic cells and cytotrophoblastic cells. She had experienced six pregnancies, and the last pregnancy ended in spontaneous abortion approximately 4 years prior. The patient was treated with etoposide, methotrexate, actinomycin D, cyclophosphamide, and vincristine (EMA/CO) 
and had suspected drug-induced pneumonia after the third course (Fig. 1). Therefore, she could not continue EMA-CO therapy although it was effective. The regimen was modified, but the new regimen proved ineffective. She was then referred to our institution for further treatment. We performed a drug-induced lymphocyte stimulation test and found that the anticancer drugs etoposide, etoposide, methotrexate, actinomycin, cyclophosphamide, and vincristine did not induce the allergy. Thus, we concluded that her pneumonia was induced by infection and that we could use these anticancer drugs. After obtaining written informed consent from the patient and her partner, we performed short tandem repeat analysis of DNA extracted from the oral mucosal cells of the patient and her partner and from the paraffin-embedded sections of the micro-dissected tumor, as previously described (5). This study was approved by the Ethics Committee of Nagoya University Graduate School of Medicine. Tumor analysis revealed gestational choriocarcinoma of both maternal and paternal origins (Table I). The patient underwent four types of chemotherapy regimens and was then treated with high-dose ifosfamide, carboplatin, and etoposide (ICE), along with autologous peripheral blood stem cell transplantation $(2,6,7)$.

After three courses of high-dose ICE, we performed total hysterectomy and bilateral adnexectomy to reduce the total choriocarcinoma volume. The patient was administered two courses of mini-ICE after the operation, but multiple metastases were found in the brain. She was thus treated with whole-brain radiotherapy (20 Gy); etoposide, cisplatin, methotrexate, and actinomycin D (EP-EMA) chemotherapy; and radiotherapy for the bone metastases.

To identify drugs appropriate for treating the choriocarcinoma in this case, we utilized the NCC Oncopanel System to compare the uterine choriocarcinoma DNA with the patient's germline DNA extracted from peripheral blood. Microdissection was performed to obtain the choriocarcinoma tissue from formaldehyde-fixed and paraffin-embedded tissue sections (10 $\mu \mathrm{m}$ thickness). The samples were prepared and analyzed as previously reported (4). NCC Oncopanel test revealed 245 single-nucleotide variants (SNVs). Compared with the usual allele frequency of SNVs in matched normaltumor pair analysis of $\leq 30 \%$, the mean SNV allele frequency of the patient was more than double, at $63.1 \%$. Initially, experimental errors such as sample misidentification were suspected; however, we eventually concluded that part of the gestational choriocarcinoma DNA was derived from the partner of the patient, whereby the SNV burden was increased.

The tumor DNA contained 19 variants in 13 of the 114 cancer-related genes (Table II). The GNAQ p.T96S and TP53 p.R213P variants were considered to be pathogenic variants; the remaining 17 variants are frequent in the Japanese population. There are no targeted therapies for these two pathogenic variants. After nine courses of EP-EMA, the patient was unable to undergo chemotherapy because of pancytopenia and febrile neutropenia. She was treated for 20 months but ultimately died of choriocarcinoma 7 months after the operation.

\section{Discussion}

This is the first study using the NCC Oncopanel System test for gestational choriocarcinoma. The test was performed to seek
Table I. Short tandem repeat analysis of DNA from the tumor, patient and her partner.

\begin{tabular}{lccc}
\hline Marker & Maternal & Paternal & Tumor \\
\hline D8S1179 & 10,14 & 13,13 & 10,13 \\
D21S11 & 30,31 & 30,30 & 30 \\
D7S820 & 11,12 & 9,12 & 9,12 \\
CFS1PO & 10,11 & 10,10 & 10 \\
D3S1358 & 16,16 & 16,17 & 16 \\
TH01 & 6,6 & 6,9 & 6 \\
D13S317 & 11,12 & 11,11 & 11,12 \\
D16S539 & 11,11 & 9,9 & 9,11 \\
D2S1338 & 17,20 & 23 & - \\
D19S433 & 13,13 & $13,15.2$ & 13 \\
vWA & 17,19 & 16,16 & $16,17,19$ \\
TPOX & 8,11 & 8,11 & 11 \\
D18S51 & 14,18 & 14,17 & 17,18 \\
Amerogenin & $\mathrm{X}, \mathrm{X}$ & $\mathrm{X}, \mathrm{Y}$ & $\mathrm{X}, \mathrm{X}$ \\
D5S818 & 10,12 & 9,11 & $10,11,12$ \\
FGA & 23,26 & 23,24 & $23,24,26$
\end{tabular}

The tumor contained maternal and paternal alleles, suggesting that it was gestational.

appropriate drugs for the intractable choriocarcinoma, but no drug matched the tumor genome. A hospital-based prospective study using the NCC Oncopanel System test showed that only $13.4 \%$ of the patients were eligible for targeted drug therapies based on the sequencing results, and this result is similar to that obtained using another cancer-gene panel (11\%) (8). The relatively low likelihood of identifying a targeted therapy should be explained to patients before applying a gene-panel test. Additional genome-matched clinical trials are required to determine the applications that these tests would suit the most.

The results of the NCC Oncopanel test in our case indicate two limitations to using NGS-based tumor-profiling multiplex gene panels for GTN patients. First, panel tests for tumor and matched non-tumor samples, such as the NCC Oncopanel test, show a high SNV burden in the genomic DNA from GTNs, and such results may be misinterpreted as 'tumor-derived' variants. The NCC Oncopanel test is inappropriate for tumors like GTNs containing the DNA of other persons. Tumor-profiling gene-testing using only tumor samples should be used for GTNs. Second, the patient, her partner, and/or their children might be the source of pathogenic variants or secondary genetic findings in the tumor DNA of GTNs. A case of choriocarcinoma in a woman whose partner had a genomic TP53 variant leading to Li-Fraumeni syndrome has been reported, wherein the TP53 variant was detected in her tumor but not in her germline DNA (9). Since there are ethical issues associated with genomic screening in GTN cases, informed consent should be obtained from patients and their partners, and specific ethical guidelines should be laid down for tumor-panel testing of patients with GTN.

In the present case, two pathogenic variants (GNAQ p.T96S and TP53 p.R213P) were identified in the tumor DNA. The 
Table II. Genomic findings for the tumor and blood of the patient, obtained using the NCC Oncopanel System Test.

\begin{tabular}{|c|c|c|c|c|}
\hline Gene name & Mutation allele frequency & Amino acid change & dbSNP & HGVD allele frequency \\
\hline BARD1 & 66.3 & $\mathrm{R} 24 \mathrm{~S}$ & rs1048108 & 0.350 \\
\hline SETD2 & 68.0 & M1080I & rs76208147 & 0.143 \\
\hline ROS 1 & 64.9 & $\mathrm{~S} 2229 \mathrm{C}$ & rs619203 & 0.145 \\
\hline ROS1 & 67.9 & K2228Q & rs529156 & 0.146 \\
\hline ROS 1 & 68.4 & $\mathrm{D} 2213 \mathrm{~N}$ & rs529038 & 0.151 \\
\hline GNAQ & 12.8 & T96S & rs777679970 & Not detected \\
\hline TP53 & 61.7 & R213P & rs587778720 & Not detected \\
\hline BRCA1 & 66.5 & $\mathrm{~S} 1613 \mathrm{G}$ & rs1799966 & 0.331 \\
\hline BRCA1 & 62.8 & K1183R & rs16942 & 0.329 \\
\hline BRCA1 & 63.0 & E1038G & rs16941 & 0.329 \\
\hline BRCA1 & 70.4 & $\mathrm{R} 871 \mathrm{~L}$ & rs799917 & 0.331 \\
\hline FGFR4 & 74.9 & G388R & rs351855 & 0.414 \\
\hline NOTCH2 & 69.0 & $\mathrm{R} 1260 \mathrm{H}$ & rs75423398 & 0.070 \\
\hline PRKCI & 73.0 & R327R & rs55683301 & 0.061 \\
\hline ESR1 & 78.5 & P146Q & rs17847065 & 0.047 \\
\hline PTCH1 & 70.1 & $\mathrm{R} 893 \mathrm{H}$ & rs138154222 & 0.019 \\
\hline BRCA2 & 62.8 & M784V & rs11571653 & 0.095 \\
\hline CREBBP & 64.6 & L551I & rs61753381 & 0.032 \\
\hline BRCA1 & 51.0 & Y856H & rs80356892 & 0.009 \\
\hline
\end{tabular}

SNP allele frequency of GNAQ and TP53 were not detected in the Japanese database, HGVD. These data demonstrated that GNAQ and TP53 may be pathogenic variants. dbSNP, database of single nucleotide polymorphism; HGVD, human genetic variation database.

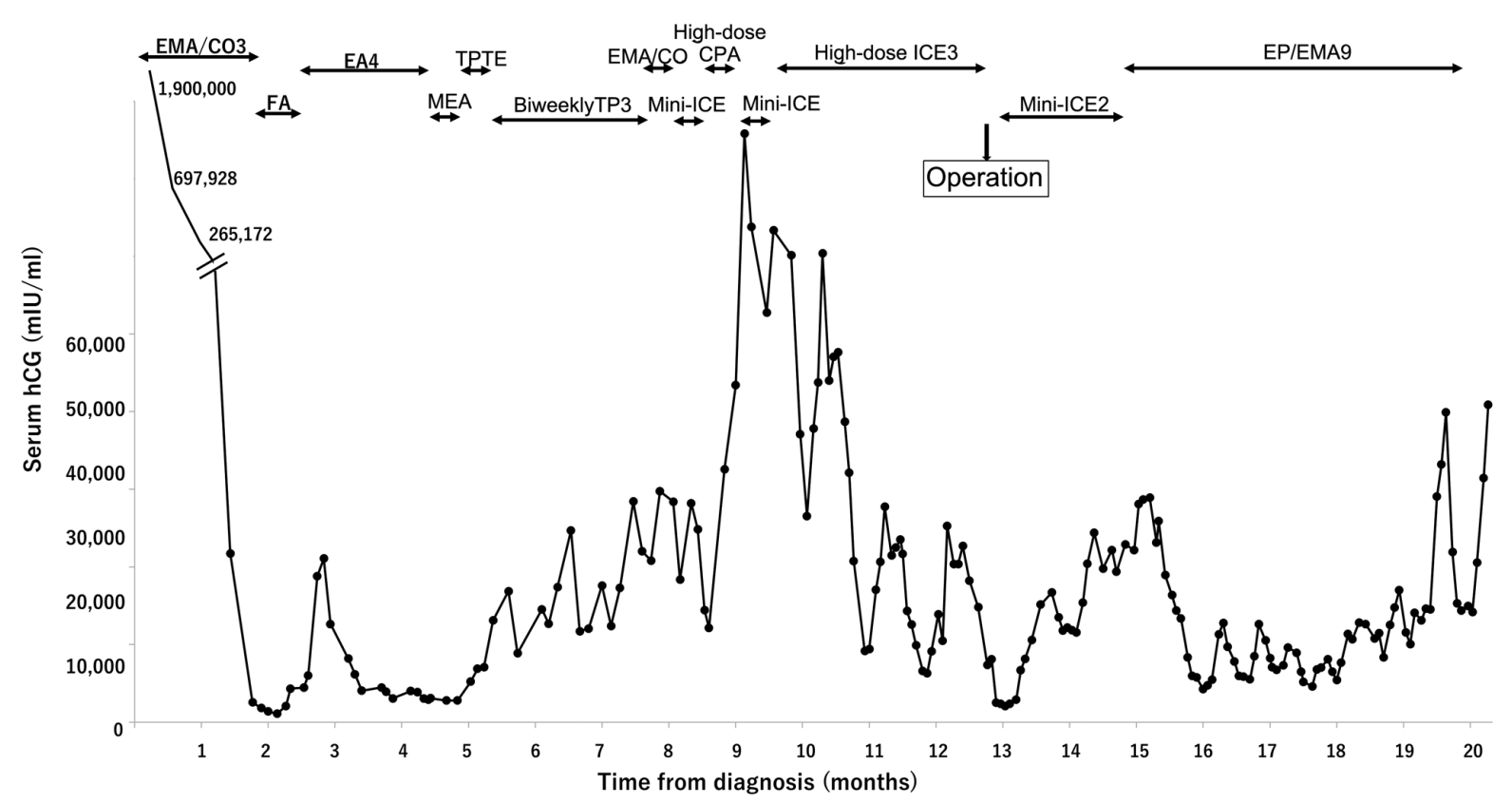

Figure 1. Changes in the serum hCG level of the patient and the treatment progress of choriocarcinoma. EMA/CO, etoposide, methotrexate, actinomycin D, cyclophosphamide, and vincristine; FA, fluorouracil and actinomycin D; EA, etoposide and actinomycin D; MEA, methotrexate, etoposide, and actinomycin D; TPTE, paclitaxel, cisplatin, and etoposide; biweekly TP, biweekly paclitaxel and cisplatin; ICE, ifosfamide, carboplatin, and etoposide; CPA, cyclophosphamide; EP/EMA, etoposide, cisplatin, methotrexate, and actinomycin D; hCG, human chorionic gonadotropin.

sequence report from the NCC Oncopanel revealed a $12.8 \%$ variant allele frequency for $G N A Q$ p.T96S, classifying this allele as a somatic variant. The variant allele frequency of TP53 p.R213P was $61.7 \%$, indicating that this allele might have been a germline variant, but the DNA of the patient's blood did not have it. We realized that this allele might have been a true somatic variant in her tumor or a germline variant in the partner, one of the children of the patient, or the lost pregnancy, because the 
tumor was a gestational choriocarcinoma. Genetic counseling sessions were conducted with the partner of the patient to discuss our findings for this variant and its association with Li-Fraumeni syndrome. Upon the request of the partner, we checked the existence of TP53 p.R213P variants with only his blood but not her children's blood. We found that he did not have this variant.

We here report a case of intractable gestational choriocarcinoma resistant to numerous chemotherapies, including high-dose ICE with peripheral stem cell rescue. It is suggested that it is difficult for choriocarcinoma patients to achieve complete remission when the second chemotherapy regimen fails and multiple metastases exist (3). High-dose chemotherapy with stem cell rescue and anti-programmed cell death-1 (PD-1) antibody therapy might be an option for intractable choriocarcinoma $(2,7,10)$. The effectiveness of anti-PD-1 antibody therapy for intractable GTN patients has recently been reported (10). Our patient was not eligible for this treatment because her choriocarcinoma did not show a high microsatellite instability status, which is required for health-insurance coverage in Japan. Clinical trials of antiPD-1 antibody therapy for intractable GTN are needed, as this therapy has been shown to be effective for patients with GTN with unknown microsatellite instability statuses (11).

In conclusion, our experience of an intractable choriocarcinoma case screened with the NCC Oncopanel System suggests that matched normal-tumor pair analysis is not appropriate for GTN. When using an NGS-based tumor panel to assess choriocarcinoma, researchers must consider whether the patient's and partner's genomic DNA is involved in the GTN.

\section{Acknowledgements}

Not applicable.

\section{Funding}

This work was financially supported by grants-in-aid numbers 17K16845 and 20K09639 (to KN) by the Ministry of Education, Culture, Sports, Science, and Technology of Japan. The funding bodies had no role in study design or data collection, analysis or interpretation.

\section{Availability of data and materials}

The datasets used and/or analyzed during the current study are available from the corresponding author on reasonable request.

\section{Authors' contributions}

KNii, EY, SM, HH, MMorik, HK and FK designed the study. KNii wrote the final manuscript. SM, HH, MMorik, MH and MMorit analyzed and interpreted the data, and wrote the outline of the manuscript regarding genetic analysis. MMorik, $\mathrm{MH}$ and MMorit were responsible for genetic counseling. KNis, YO, EW and TY analyzed and interpreted the data of STR analysis. KNii, KNis, YO, EW, HK and FK were involved in the treatment of patients as attending physicians and provided important advice for decision making. EY, TY, HK and FK critically revised the paper for important intellectual content. KNii and EY confirmed the authenticity of all the raw data. All authors read and approved the final manuscript.

\section{Ethics approval and consent to participate}

Written informed consent was obtained from the study subjects prior to the collection of all the biological samples according to the regulations set out by the Ethics Committee at Nagoya University.

\section{Patient consent for publication}

Written informed consent was obtained from the partner of the patient for the publication of these data.

\section{Competing interests}

The authors declare that they have no competing interests.

\section{References}

1. Albrecht C, Chamley L, Charnock-Jones DS, Collins S, Fujiwara H, Golos T, Grayo S, Hannan N, Harris L, Ichizuka K, et al: IFPA meeting 2018 workshop report II: Abnormally invasive placenta; inflammation and infection; preeclampsia; gestational trophoblastic disease and drug delivery. Placenta 84: 9-13, 2019.

2. Yamamoto E, Niimi K, Fujikake K, Nishida T, Murata M, Mitsuma A, Ando Y and Kikkawa F: High-dose chemotherapy with autologous peripheral blood stem cell transplantation for choriocarcinoma: A case report and literature review. Mol Clin Oncol 5: 660-664, 2016.

3. Powles T, Savage PM, Stebbing J, Short D, Young A, Bower M, Pappin C, Schmid P and Seckl MJ: A comparison of patients with relapsed and chemo-refractory gestational trophoblastic neoplasia. Br J Cancer 96: 732-737, 2007.

4. Sunami K, Ichikawa H, Kubo T, Kato M, Fujiwara Y, Shimomura A, Koyama T, Kakishima H, Kitami M, Matsushima $\mathrm{H}$, et al: Feasibility and utility of a panel testing for 114 cancer-associated genes in a clinical setting: A hospital-based study. Cancer Sci 110: 1480-1490, 2019.

5. Yamamoto E, Niimi K, Shinjo K, Yamamoto T, Fukunaga M and Kikkawa F: Identification of causative pregnancy of gestational trophoblastic neoplasia diagnosed during pregnancy by short tandem repeat analysis. Gynecol Oncol Case Rep 9: 3-6, 2014.

6. Piamsomboon S, Kudelka AP, Termrungruanglert W, Van Besien K, Edwards CL, Lifshitz S, Schomer DF, Champlin R, Mante RP, Kavanagh JJ and Verschraegen CF: Remission of refractory gestational trophoblastic disease in the brain with ifosfamide, carboplatin, and etoposide (ICE): First report and review of literature. Eur J Gynaecol Oncol 18: 453-456, 1997.

7. van Besien K, Verschraegen C, Mehra R, Giralt S, Kudelka AP, Edwards CL, Piamsonboom S, Termrungruanglert W, Champlin R and Kavanagh JJ: Complete remission of refractory gestational trophoblastic disease with brain metastases treated with multicycle ifosfamide, carboplatin, and etoposide (ICE) and stem cell rescue. Gynecol Oncol 65: 366-369, 1997.

8. Zehir A, Benayed R, Shah RH, Syed A, Middha S, Kim HR, Srinivasan P, Gao J, Chakravarty D, Devlin SM, et al: Mutational landscape of metastatic cancer revealed from prospective clinical sequencing of 10,000 patients. Nat Med 23: 703-713, 2017.

9. Cotter JA, Szymanski L, Karimov C, Boghossian L, Margol A, Dhall G, Tamrazi B, Varaprasathan GI, Parham DM, Judkins AR and Biegel JA: Transmission of a TP53 germline mutation from unaffected male carrier associated with pediatric glioblastoma in his child and gestational choriocarcinoma in his female partner. Cold Spring Harb Mol Case Stud 4: a002576, 2018.

10. Choi MC, Oh J and Lee C: Effective anti-programmed cell death 1 treatment for chemoresistant gestational trophoblastic neoplasia. Eur J Cancer 121: 94-97, 2019.

11. Ghorani E, Kaur B, Fisher RA, Short D, Joneborg U, Carlson JW, Akarca A, Marafioti T, Quezada SA, Sarwar N and Seckl MJ: Pembrolizumab is effective for drug-resistant gestational trophoblastic neoplasia. Lancet 390: 2343-2345, 2017.

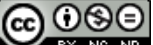

This work is licensed under a Creative Commons Attribution-NonCommercial-NoDerivatives 4.0 International (CC BY-NC-ND 4.0) License. 